\title{
Simulation of Time-Multiplexing Cellular Neural Networks with Numerical Integration Algorithms
}

\author{
V. Murugesh and K. Murugesan \\ Department of Computer Science and Engineering, \\ National Institute of Technology, Tiruchirappalli - 620 015, Tamil Nadu, India \\ \{murugesh, murugu\} @nitt. edu
}

\begin{abstract}
A novel approach to simulate Cellular Neural Networks (CNN) is presented in this paper. The approach, time-multiplexing simulation, is prompted by the need to simulate hardware models and test hardware implementations of CNN. For practical applications, due to hardware limitations, it is impossible to have a one-to-one mapping between the CNN hardware processors and all the pixels of the image. This simulator provides a solution by processing the input image block by block, with the number of pixels in a block being the same as the number of CNN processors in the hardware. The algorithm for implementing this simulator is presented along with popular numerical integration algorithms. Some simulation results and comparisons are also presented.
\end{abstract}

\section{Introduction}

Cellular Neural Networks (CNNs) are analog, time-continuous, nonlinear dynamical systems and formally belong to the class of recurrent neural networks. Since their introduction in 1988 (by Chua and Yang [2-3]), it has been the subject of intense research. Initial applications include image processing, signal processing, pattern recognition and solving partial differential equations etc.

Lee and Pineda de Gyvez [4] introduced Euler, Improved Euler and Fourth-Order Runge-Kutta algorithms in time-multiplexing $\mathrm{CNN}$ simulation. In this article, we consider the same problem (discussed by Chi-Chien Lee and Jose Pineda de Gyvez [4]) but presenting a different approach using the algorithms such as Euler, RK-Gill and RK-Butcher with more accuracy.

\section{Cellular Neural Networks}

The basic circuit unit of $\mathrm{CNN}$ is called a cell. It contains linear and nonlinear circuit elements. Any cell, $C(i, j)$, is connected only to its neighbouring cells i.e. adjacent cells interact directly with each other. This intuitive concept is called neighbourhood and is denoted as $N(i, j)$. Cells not in the immediate neighbourhood have indirect effect because of the propagation effects of the dynamics of the network. Each cell has a state $x$, input $u$, and output $y$. The state of each cell is bounded for all time $t>0$ and, after the transient has settled down, a cellular 
neural network always approaches one of its stable equilibrium points. This fact is relevant because it implies that the circuit will not oscillate. The dynamics of a CNN has both output feedback (A) and input control (B) mechanisms. The first order nonlinear differential equation defining the dynamics of a cellular neural network cell can be written as follows

$$
\begin{gathered}
C \frac{d x_{i j}}{d t}=-\frac{1}{R} x_{i j}(t)+\sum_{C(k, l) \in N(i, j)} A(i, j ; k, l) y_{k j}(t)+\sum_{C(k, l) \in N(i, j)} B(i, j ; k, l) u_{k l}+I \\
y_{i j}(t)=\frac{1}{2}\left(\left|x_{i j}(t)+1\right|-\left|x_{i j}(t)-1\right|\right)
\end{gathered}
$$

where $x_{i j}$ is the state of cell $C(i, j), x_{i j}(0)$ is the initial condition of the cell, $C$ is a linear capacitor, $R$ is a linear resistor, $I$ is an independent current source, $A(i, j ; k, l) y_{k l}$ and $B(i, j ; k, l) u_{k l}$ are voltage controlled current sources for all cells $C(k, l)$ in the neighbourhood $N(i, j)$ of cell $C(i, j)$, and $y_{i j}$ represents the output equation.

It is to be noted from the summation operators that each cell is affected by its neighbour cells. $A($.$) acts on the output of neighbouring cells and is referred to as the$ feedback operator. $B($.$) in turn affects the input control and is referred to as the control$ operator. Specific entry values of matrices $A($.$) and B($.$) , are application dependent,$ are space invariant and are called cloning templates. A current bias $I$ and the cloning templates determine the transient behavior of the cellular nonlinear network.

For software simulation purposes, equation (1) is solved within each cell, in a discretized form, to simulate its state dynamics. One common way of processing a large complex image is using a raster $\mathrm{CNN}$ approach discussed by Murugesh and Murugesan [7]. This approach implies that each pixel of the image is mapped onto a CNN processor. That is, we have an image processing function in the spatial domain that can be expressed as:

$$
g(x, y)=T(f(x, y))
$$

where $f($.$) is the input image, g($.$) the processed image, and T$ is an operator on $f($. defined over the neighbourhood of $(x, y)$. From the hardware implementation's point of view, this is a very exhaustive approach. For practical applications, in the order of 2,50,000 pixels, the hardware would require an enormous amount of processors which would make its implementation unfeasible. An alternative to this scenario is time-multiplexing simulation.

\section{Time-Multiplexing Simulation}

Image sizes may be in the order of thousands of pixels which mean a large CNN array is required. This is because the mapping between the image and CNN is one-to-one and each pixel in the image has a corresponding cell in the CNN array. Practically this is unfeasible. Multiplexing the image processing operator is a suitable method to overcome this problem. In this approach, the image is 
processed block by block and the block size is equal to the used CNN array dimension. This approach leads to two errors in the calculation of border pixels of any block since they are calculated without the effect of their neighbours. The following two equations state these errors using a neighbourhood radius equal to one:

$$
\begin{aligned}
\varepsilon_{i j}^{B} & =\sum_{i=1}^{i=3} b_{i j+1} \operatorname{sign}\left(u_{i j+1}\right) \\
\varepsilon_{i j}^{A} & =\sum_{i=1}^{i=3} a_{i j+1} y_{i j+1}(t)
\end{aligned}
$$

Equation (4) represents the error $\varepsilon_{i j}^{A}$ caused in the calculation of a border cell $C_{i j}$ in the current block due to loosing the feedback effect of neighbouring cells and neglecting the feed-forward effect. Equation (3) represents the error $\varepsilon_{i j}^{B}$ caused in the calculation of a border cell $C_{i j}$ due to loosing the feed-forward effect of its neighbouring cells and neglecting the feedback effect. Both the equations (3) and (4) are written for two horizontally adjacent blocks.

\subsection{Overlap and Belt Approaches}

To calculate the border cells more accurately, the following two approaches are used:

(i) To eliminate the error $\mathcal{E}_{i j}^{B}$, a belt of width equal to the neighbourhood radius of $\mathrm{CNN}$ from the original image is used around the block, as shown in the Fig. 1(a).

(ii) An overlap between every two adjacent blocks block $_{i}$ and block $_{i+1}$ is used to minimize the error $\varepsilon_{i j}^{A}$, as shown in Fig. 1(b). This overlap is proportional to twice the neighbourhood radius of the CNN. The result of simulating block $_{i}$ is stored except the outer row or column in the overlap area between the two blocks that belongs to block $_{i+1}$ is set to the final state of block $k_{i}$.

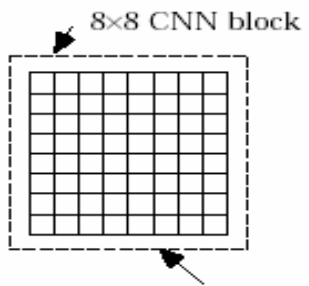

Belt of inputs $(10 \times 10$ array)

(a)

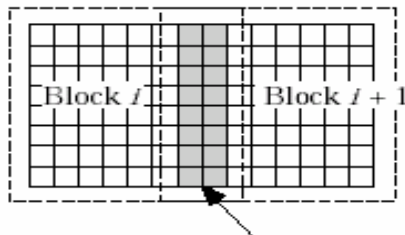

Overlapped cells

(b)

Fig. 1. (a) Belt of inputs (b) Overlapped pixels 
For the purpose of understanding the overall idea of this simulation approach better, the simplified algorithm is presented below:

Algorithm: (Time-Multiplexing CNN simulation)

$B=\left(C_{i j} \backslash i=1, \ldots\right.$, block $_{-} x^{\wedge} j=1, \ldots$, block $\left._{-} y\right)$

$P \subset B=$ set of border cells (lower left corner)

overlap = number of cell overlaps

belt = width of input cells

$\mathrm{M}=$ number of rows of the image

$\mathrm{N}$ = number of columns of the image

for $\left(i=0 ; i<M ; I+=b l o c k \_x-o v e r l a p\right)$

for $\left(j=0 ; j<N ; j+=b l o c k \_y-o v e r l a p\right)$

/* load initial conditions for the cells in the block except for those in the borders */

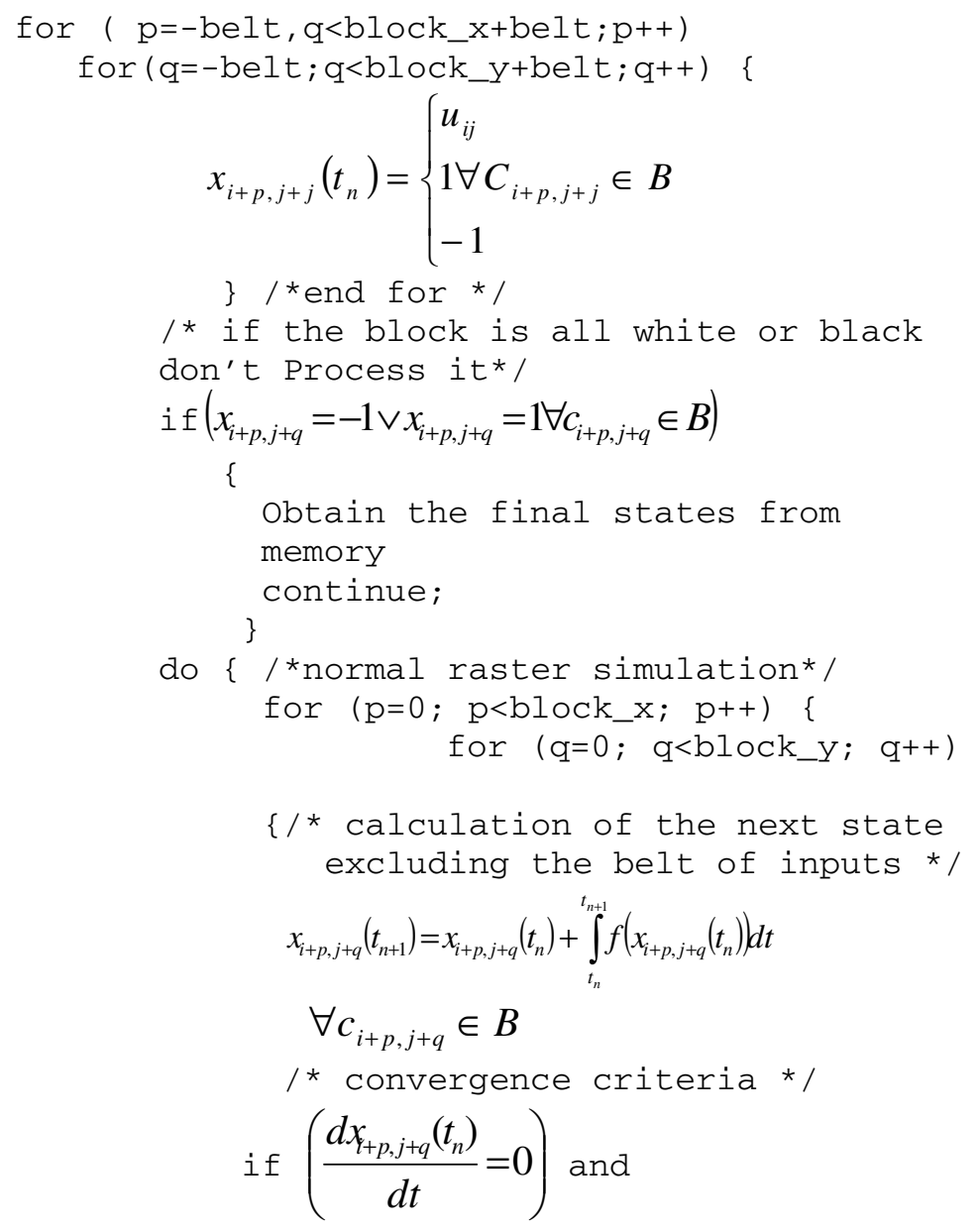




$$
\begin{aligned}
& y_{k l}= \pm 1 \\
& \forall C(k, l) \in N_{r}(i+p, j+q)\{ \\
& \text { converged_cellst+; } \\
& \text { \} } / * \text { end for * / } \\
& \text { /* update the state values */ } \\
& x_{i+p, j+q}\left(t_{n}\right)=x_{i+p, j+q}\left(t_{n}+1\right) \forall c_{i+p, j+q} \in B \text {; } \\
& \text { \}while (converged_cells<(block_x*blovk_y)) ; } \\
& \text { /* store new state values excluding the ones } \\
& \text { corresponding to the border cells* / } \\
& A \leftarrow x_{i j} \forall C_{i j} \in B \backslash P \\
& \text { \} } / \text { *end for */ }
\end{aligned}
$$

\section{Numerical Integration Algorithms}

The CNN is described by a system of nonlinear differential equations. Therefore, it is necessary to discretize the differential equation for performing simulations. For computational purpose, a normalized time differential equations describing $\mathrm{CNN}$ is used by Nossek et al. [10].

$$
\begin{aligned}
& f^{\prime}(x(\pi \tau)):=\frac{d x_{i j}(\pi \tau)}{d t}=-x_{i j}(\pi \tau)+\sum_{C(k, l) \in N_{r}(i, j)} A(i, j ; k, l) y_{k l}(\pi \tau) \\
& +\sum_{C(k, l) \in N_{r}(i, j)} B(i, j ; k, l) u_{k l}+I \\
& y_{i j}(\pi \tau)=\frac{1}{2}\left(\left|x_{i j}(\pi \tau)+1\right|-\left|x_{i j}(\pi \tau)-1\right|\right)
\end{aligned}
$$

Where $\tau$ is the normalized time. For the purpose of solving the initial-value problem, well established numerical integration techniques are used. These methods can be derived using the definition of the definite integral

$$
x_{i j}((n+1) \tau)-x_{i j}(\pi \tau)=\int_{\tau_{n}}^{\tau_{n+1}} f^{\prime}(x(\pi \tau)) d(\pi \tau)
$$

Three of the most widely used Numerical Integration Algorithms are used in Time-Multiplexing Simulation described here. They are the Euler's Algorithm; RK-Gill Algorithm discussed by Oliveira [9] and the RK-Butcher Algorithm discussed by Murugesan et al. [5], [6] and Murugesh and Murugesan [7], [8].

\section{Simulation Results and Comparisons}

All the simulation reported here are performed using a SUN BLADE 1500 workstation, and the simulation time used for comparisons is the actual CPU time used. 
The input image format is the bitmap format (xbm), which is commonly available and easily convertible from popular image formats like GIF or JPEG.

Using actual numbers can easily show how much improvement is achieved. The size of Fig. 2(a) is $355 \times 400$ (1,42,000 pixels), and an Averaging template is used for simulation comparisons. First, using the raster CNN simulator discussed by Murugesh and Murugesan [7], the simulation took 200.42 seconds. Next, with the regular timemultiplexing simulator (with overlapping and input belt) the simulation took 342.28 seconds. Finally, the time-multiplexing with the time-saving scheme performed the same simulation in 240.20 seconds, almost a $33 \%$ improvement from the regular time-multiplexing. The size of two dimensional window of $10 \times 10$, with two column overlapping is used. It may be noted that this algorithm maintains all the edges of the original one.

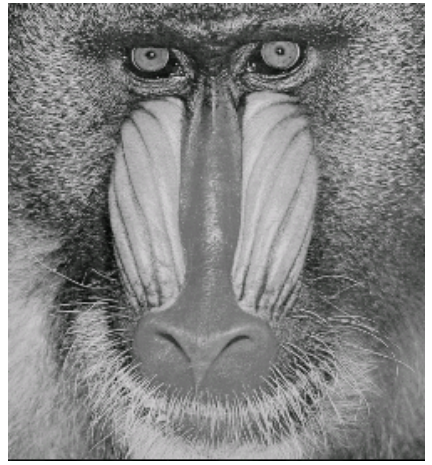

(a)

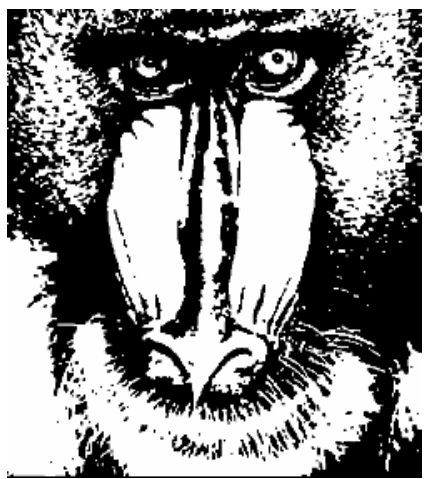

(b)

Fig. 2. (a) Original image (b) After Averaging Template using Time-Multiplexing simulation

Since speed is one of the main concerns in the simulation, finding the maximum step size that still yields convergence for a template can be of helpful in speeding up the system. The speed-up can be achieved by selecting an appropriate step size $\Delta t$ for that particular template. Even though the maximum step size may slightly vary from one image to another, the values in the Fig. 3 serve as a good reference for step size comparison. These results were obtained by trial and error over more than 100 simulations on a diamond figure. If the step size is chosen too small, the simulation might take much iteration; hence, it will take longer time to achieve convergence.

On the other hand, if the step size is taken too large, it might not converge at all or it would converge to erroneous steady state values (beyond step size 5); the latter remark can be observed for the Euler and RK-Gill algorithm which is plotted in Fig. 4. Hence, the speed of convergence of RK-Butcher algorithm for large step size is much faster than Euler and RK-Gill algorithms. 


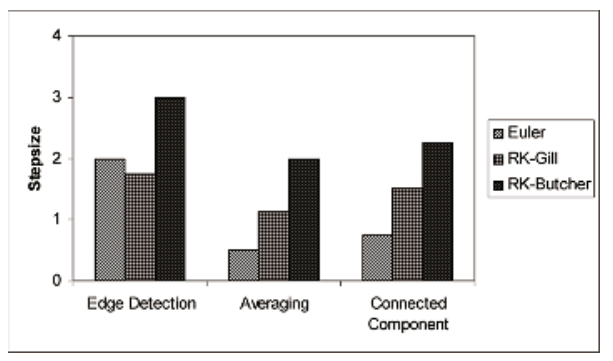

Fig. 3. Maximum step size for three different templates

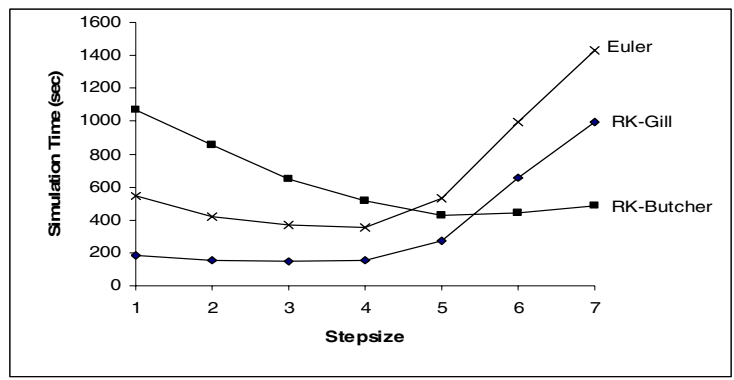

Fig. 4. Simulation time comparison using Edge Detection template

The results of Fig. 4 were obtained by simulating a small image of size $16 \times 16$ (256 pixels) using Edge Detection template on a diamond figure.

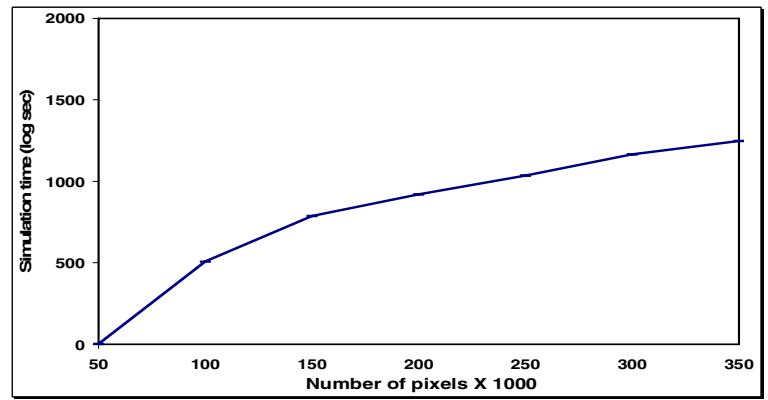

Fig. 5. CPU performance (SUN BLADE 1500 work station) for distinct sizes in number of pixels

Simulation time computations are shown in Fig. 5 using an Averaging template for images of sizes about 2,50,000 pixels and it is observed from Fig. 5, the simulation time will increase if the number of pixels chosen is too large in a log linear fashion. 


\section{Conclusion}

Versatile algorithms have been developed using numerical integration algorithms for simulating CNN with Time-Multiplexing scheme. This Time Multiplexing algorithm is a very simple one and a powerful method for developing image processors using $\mathrm{CNN}$. In fact, among all the three numerical integration algorithms, the one developed using RK-Butcher algorithm is performing very efficiently for solving this problem. The notable observation is Time Multiplexing CNN with Numerical Integration always converges for a larger step size, which in turn results in lowest simulation time for any given image size. For a given step size, the convergence time of this algorithm is log linear for all larger size images and this is an additional attractive feature as we deal with high resolution/ large images. This algorithm always preserves the edges as given in the original in addition and it enhances the picture quality.

\section{References}

1. Butcher, J. C.: The Numerical Methods of Ordinary Differential Equations, John Wiley \& Sons, U.K. (2003).

2. Chua, L. O., and Yang, L.: Cellular Neural Networks: Theory, IEEE Transactions on Circuits and Systems, 35 (1988) 1257 - 1272.

3. Chua, L. O., and Yang, L.: Cellular Neural Networks: Applications, IEEE Transactions on Circuits and Systems, 35 (1988) 1273 - 1290.

4. Chi-Chien Lee and Jose Pineda de Gyvez: Time-Multiplexing CNN Simulator, IEEE International Symposium on Circuits and Systems, 6 (1999) 407-410.

5. Murugesan, K., Sekar, S., Murugesh, V. and Park, J. Y.: Numerical Solution of an Industrial Robot Arm Control Problem using the RK-Butcher algorithm, International Journal of Computer Applications in Technology, 19 (2004) 132-138.

6. Murugesan, K., Gopalan, N. P., and Devarajan Gopal.: Error free Butcher algorithms for linear Electrical Circuits, ETRI Journal, 27 (2005) 195-205.

7. Murugesh, V., and Murugesan, K.: Comparison of Numerical Integration Algorithms in Raster CNN Simulation, Lecture Notes in Computer Science, Vol. 3285, Springer-Verlag, Berlin Heidelberg New York (2004), pp. 115-122.

8. Murugesh,V. and Murugesan, K.: Simulation of Cellular Neural Networks using the RKButcher algorithm, International Journal of Management and Systems, 21(2005)65-78.

9. Oliveira, S. C.: Evaluation of effectiveness factor of immobilized enzymes using RungeKutta-Gill method: how to solve mathematical undetermination at particle center point?", Bio Process Engineering, 20 (1999) 185-187.

10. Nossek, J. A., Seiler, G., Roska. T., and Chua. L. O.: Cellular Neural Networks: Theory and Circuit Design, International Journal of Circuit Theory and Applications, 20 (1992) 533-553. 